\title{
Suppression of SCC antigen promotes cancer cell invasion and migration through the decrease in E-cadherin expression
}

\author{
AKIHIRO MURAKAMI, TATSUSHI NAKAGAWA, MAYUMI KANEKO, SHUGO NAWATA, \\ OSAMU TAKEDA, HIROSHI KATO and NORIHIRO SUGINO \\ Department of Obstetrics and Gynecology, Yamaguchi University Graduate \\ School of Medicine, 1-1-1 Minamikogushi, Ube 755-8505, Japan
}

Received May 5, 2006; Accepted July 3, 2006

\begin{abstract}
Squamous cell carcinoma antigen (SCCA) is a useful tumor marker for diagnosis and management of squamous cell carcinoma. Recent studies have shown that SCCA can influence the behavior of cancer cells. It is well known that cell-cell adhesion is an important factor for the progression of cancer. The present study, therefore, was undertaken to investigate the effect of SCCA2 on the cell adhesion related molecule, E-cadherin, and cancer cell behavior. For this purpose, antisense SCCA2 cDNA was transfected into human uterine cancer cell lines, SKG IIIa and SiHa, which express SCCA2. Suppression of SCCA2 expression by antisense $S C C A 2$ cDNA transfection decreased E-cadherin expression and promoted cell migration and invasion as well as the blockage of E-cadherin function by anti-E-cadherin antibody administration. In conclusion, SCCA2 regulates cell migration and invasion via E-cadherin expression, suggesting that SCCA2 may be involved in cancer behavior such as invasion or metastasis.
\end{abstract}

\section{Introduction}

Squamous cell carcinoma antigen (SCCA) was first discovered in uterine cervical squamous cell carcinoma by Kato and Torigoe (1), and was widely used as a serological tumor marker for diagnosis and management of squamous cell carcinoma (2-4). Recent molecular studies have revealed that SCCA is encoded by two highly homologous genes, SCCA1 and SCCA2, at 18q21.3 locus $(5,6)$. Interestingly, both SCCA1 and SCCA2 belong to the serine proteinase inhibitor (serpin) family $(5,7)$. SCCA1 inhibits both serine proteinase and cysteine proteinase, while SCCA2 inhibits serine proteinase, chymase and cathepsin $\mathrm{G}(8-11)$. These facts make us speculate that

Correspondence to: Dr Norihiro Sugino, Department of Obstetrics and Gynecology, Yamaguchi University Graduate School of Medicine, 1-1-1 Minamikogushi, Ube 755-8505, Japan

E-mail: sugino@yamaguchi-u.ac.jp

Key words: SCC antigen, E-cadherin, cell migration and invasion
SCCA may have important biological functions in squamous cell carcinoma. In fact, our previous studies have shown that SCCA serves as a survival factor against apoptosis in human cancer cells, suggesting a possibility that SCCA may be involved in the regulation of cancer behaviors $(12,13)$.

A number of studies have shown that altered expression of cell adhesion molecules is involved in cancer behaviors, especially in the process of invasion and metastasis. Loss of E-cadherin expression, a main cell adhesion molecule of epithelium that mediates cell to cell contacts, causes the detachment of cancer cells in the primary tumor, which is the first step of cancer metastasis (14). SCCA is expressed not only in squamous cell carcinoma but also in normal squamous epithelium (15), and SCCA mRNA expression is mainly observed in the basal and parabasal layers of normal squamous epithelium by in situ hybridization (16). It is of interest to note that E-cadherin is also expressed in the basal and parabasal layers (17). These findings strongly suggest a possibility that SCCA may be closely related with E-cadherin expression. Therefore, it is interesting to study a possibility that SCCA may be involved in cancer behavior such as invasion or metastasis through alteration in E-cadherin expression.

\section{Materials and methods}

Cell culture. Human uterine cervical cancer cell line, SKG IIIa, which expresses SCCA, was kindly provided by Dr S. Nozawa (Keio University, Tokyo, Japan) (18). This cell line was grown in Ham's F 12 (Sigma, Saint Louis, USA) supplemented with $5 \%$ fetal calf serum (FCS), $100 \mathrm{U} / \mathrm{ml}$ penicillin, and $100 \mathrm{mg} / \mathrm{ml}$ streptomycin at $37^{\circ} \mathrm{C}$ in a humidified $5 \% \mathrm{CO}_{2}$ incubator. SiHa, which also expresses SCCA, was obtained from Cell Resource Center for Biomedical Research (Tohoku University, Sendai, Japan). This cell line was cultured in Eagle's minimal essential medium (Sigma) with the same condition of SKG IIIa cells.

Construction of the antisense expression vector and gene transfection. To analyze the function of SCCA2, we established stable clones whose SCCA2 expression was decreased. To suppress the expression of SCCA2, transfection of the antisense construct or the empty vector to uterine squamous cell carcinoma cells was performed as reported previously (12). Suppression of SCCA2 was confirmed by Western blot analysis as described below. 


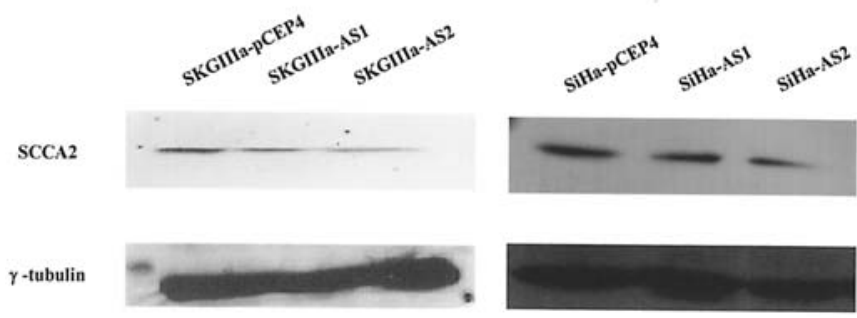

Figure 1. Establishment of clones with decreased SCCA2 expression. Antisense cDNA to SCCA2 was transfected into a human uterine cervical cancer cell line, SKG IIIa and SiHa. Two independent clones in which SCCA2 expression was suppressed and a control clone were obtained. Expression levels of SCCA2 in the transfected cells were examined by Western blotting. (A) SKG IIIa cells transfected with antisense SCCA2 cDNA (SKG IIIa-AS1, SKG IIIa-AS2) or the empty vector (control; SKG IIIa-pCEP4). (B) SiHa cells transfected with antisense SCCA2 cDNA (SiHa-AS1, SiHa-AS2) or the empty vector (control; SiHa-pCEP4). Data are representatives of three independent experiments. $\gamma$-tubulin is used as an internal control.

Western blot analysis. To determine the inhibition of SCCA2 expression and the change of expression level of E-cadherin, Western blot analyses were performed. Equal amounts of total protein were electrophoresed to SDS-PAGE. After SDS-PAGE was completed, proteins were transferred to the polyvinylidene difluoride membrane (ATTO, Tokyo, Japan) with semi-dry type blotting system. The transferred membranes were stained by immunochemical technique consisting of the following procedure: after blocking the membrane with blocking solution (5\% skimmed milk with $0.1 \%$ Tween-20 dissolved in Trisbuffered saline, $\mathrm{pH}$ 7.5), the blotted membranes were incubated with the first antibody [the monoclonal antibody against SCCA2 kindly presented by Dr Gary A. Silverman and monoclonal antibodies against E-cadherin (Santa Cruz Biotechnology, USA) or $\gamma$-tubulin (Sigma)], which were diluted in blocking solution. Then, these membranes were incubated with the peroxidase conjugated second antibody diluted in blocking solution. Finally, an ECL-Western blotting detection system (Amersham, Aylesburg, UK) was applied according to the manufacturer's protocol and then the membranes were exposed to hyperfilm-ECL (Amersham).

Assay for cell invasion and cell migration. To analyze the change of cell functions caused by SCCA2 suppression, cell migration and cell invasion were examined as reported previously (19). Briefly, the cell culture was performed using 24-well culture plates. An insert was placed into each well of the plate, effectively creating a lower and upper chamber. The bottom of the insert was made of $8-\mu \mathrm{m}$ pore size polycarbonate membranes which were either coated with or without Matrigel (Becton Dickinson BioCoat, NJ, USA). The cells were then cultured in the culture medium without FCS at a density of $5 \times 10^{4}$ cells/well in the upper chamber and the lower chamber was filled with culture medium supplemented with 5\% FCS. After incubation for $21 \mathrm{~h}$, the cells on the upper surface of the membranes were completely removed by wiping with cotton swabs. The filters were fixed and stained with DiffQuick (Sysmex, Kobe, Japan). The cells on the lower surface were counted at magnification x200 in 5 randomized field views. Next, numbers of migrating cells were counted on the lower surface of the non-Matrigel coated chamber. Percentages of invading cells were calculated by dividing the number of cells in Matrigel by the number of invading cells in the nonMatrigel. Each experiment was performed in triplicate wells. Mean percentages were obtained from results from the triplicate and three different experiments were performed.

Blockage of E-cadherin function. To analyze the effect of the blockage of E-cadherin on cell invasion and migration, antiE-cadherin antibody was added as reported previously (20). Cancer cells were cultured at a density of $5 \times 10^{4}$ cells/well in the upper chamber with or without $1.0 \mu \mathrm{g} / \mathrm{ml}$ of anti-E-cadherin antibody under the same condition described above (Alexis Biochemicals, Lausen, Switzerland). Assays for cell invasion and cell migration were performed as described above.

Statistical analysis. Statistical analysis was performed by Duncan's new multiple range test. A probability value of $\mathrm{p}<0.05$ was considered to be significant.

\section{Results}

Establishment of clones with decreased SCCA2 expression. To examine the effect of SCCA2 on E-cadherin and cancer cell invasion and migration, antisense SCCA2 cDNA was transfected into SKG IIIa and SiHa cells. We obtained two independent clones less-expressing SCCA2 (SKG IIIa-AS1, -AS2 and SiHa-AS1, -AS2, respectively). It was confirmed by Western blotting that the expression of SCCA2 was suppressed in both SKG IIIa-AS1 and -AS2 clones and SiHa-AS1 and -AS2 clones compared to those of the control (SKG IIIapCEP4 and SiHa-pCEP4) (Fig. 1).

Effects of SCCA2 suppression on E-cadherin expression. To study the effect of SCCA2 suppression on E-cadherin expression, we examined E-cadherin expression in the antisense SCCA2 transfectants and the control cells. In the clones of SKG IIIa-AS1, -AS2 or SiHa-AS1, -AS2, expression of E-cadherin was lower compared with the control cells (SKG IIIa-pCEP4 and SiHa-pCEP4) (Fig. 2).

Effects of SCCA2 suppression on cell invasion activity. To examine the effect of SCCA2 suppression on the cell invasion activity, we observed \% cell invasion in the antisense SCCA2 transfectants and the control cells. There was a significant difference in \% cell invasion between the antisense transfectants (SKG IIIa-AS1, -AS2) and the control cells (SKG IIIa-pCEP4), but there was no difference between SKG IIIa-AS1 and SKG IIIa-AS2 (Fig. 3A). Although invasion activity in SiHa cells was lower than that in SKG IIIa cells, a significant difference in \% cell invasion was also found between the antisense transfectants (SiHa-AS1, -AS2) and the control cells (SiHa-pCEP4) (Fig. 3B). There was also no difference between SiHa-AS1 and SiHa-AS2 (Fig. 3B).

Effects of blockage of E-cadherin function on cell invasion activity. To examine the effect of blockage of E-cadherin function on the cell invasion activity, we observed \% cell invasion in the parent cell (SKG IIIa and $\mathrm{SiHa}$ ) with or without anti-E-cadherin antibody. The cell invasion activity was 


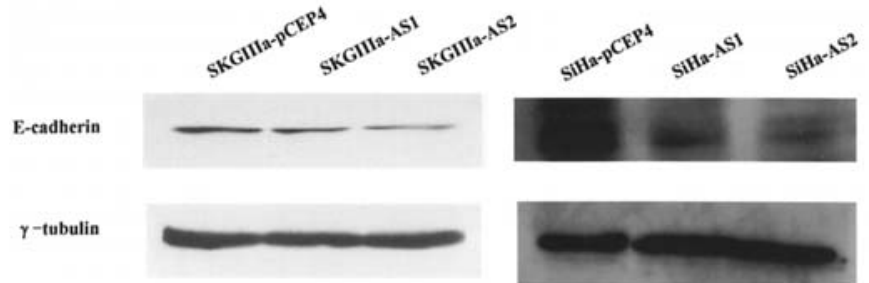

Figure 2. Effect of SCCA2 suppression on E-cadherin expression. Expression levels of E-cadherin were examined by Western blotting in the cells shown in the legend to Fig. 1. (A) SKG IIIa cells transfected with antisense SCCA2 cDNA or the empty vector. (B) SiHa cells transfected with antisense SCCA2 cDNA or the empty vector. Data are representatives of three independent experiments. $\gamma$-tubulin is used as an internal control.
A

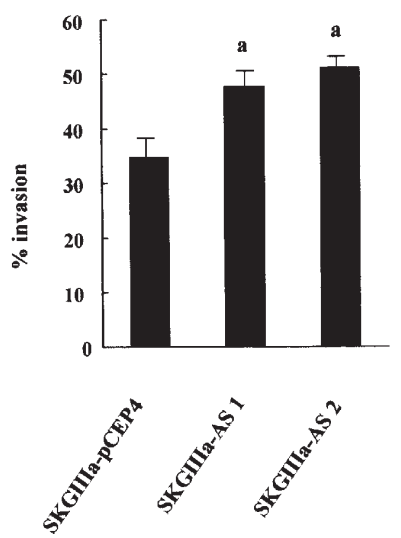

B

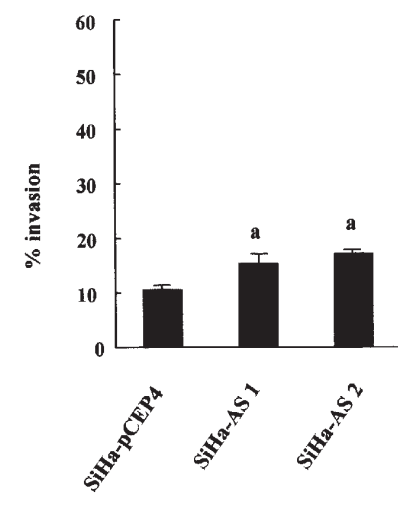

Figure 3. Effects of SCCA2 suppression on cell invasion activity. Invasion activity of SKG IIIa and SiHa transfected with antisense SCCA2 or an empty vector was expressed as \% cell invasion, as described in Materials and methods. The invaded and migrated cells were counted under a phase contrast microscope. Percentage cell invasion is shown as mean \pm SD of three independent experiments. (A) SKG IIIa cells transfected with antisense SCCA2 cDNA (SKG IIIa-AS1, SKG IIIa-AS2) or the empty vector (control; SKG IIIa-pCEP4). (B) SiHa cells transfected with antisense SCCA2 cDNA (SiHa-AS1, SiHa-AS2) or the empty vector (control; SiHapCEP4). ${ }^{a} \mathrm{p}<0.05$ vs. control.

significantly increased by anti-E-cadherin antibody treatment in both SKG IIIa cells (Fig. 4A) and SiHa cells (Fig. 4B).

Effects of suppression of SCCA2 on cell migration activity. To study the effect of SCCA2 suppression on the cell migration activity, we examined the ratio of antisense SCCA2 transfectants to control cells in the non-coated chambers. Cell migration activity in the antisense SCCA2 transfectants (SKG IIIa-AS1, -AS2) was significantly higher than that of the control cells (SKG IIIa-pCEP4) (Fig. 5A). In SiHa cells, the migration activity in the antisense SCCA2 transfectants (SiHaAS1, -AS2) was also significantly higher than that of the control cells (SiHa-pCEP4) (Fig. 5B).

Effects of blockage of E-cadherin function on cell migration activity. To examine the effect of blockage of E-cadherin function on the cell migration activity, we observed the ratio
A
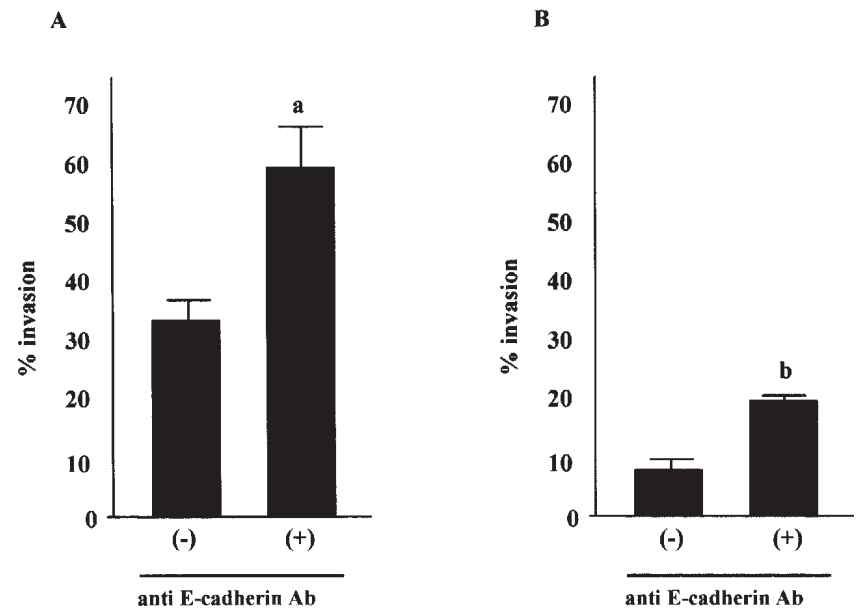

Figure 4. Effects of blockage of E-cadherin function on cell invasion activity. Invasion activity of SKG IIIa and SiHa with or without anti-E-cadherin antibody was expressed as \% cell invasion, as described in Materials and methods. The invaded and migrated cells were counted under a phase contrast microscope. Percentage cell invasion is shown as mean $\pm \mathrm{SD}$ of three independent experiments. (A) SKG IIIa (B) $\mathrm{SiHa}{ }^{\mathrm{a}} \mathrm{p}<0.01$; and ${ }^{\mathrm{b}} \mathrm{p}<0.05$ vs. anti-E-cadherin $\mathrm{Ab}(-)$.
$\mathbf{A}$

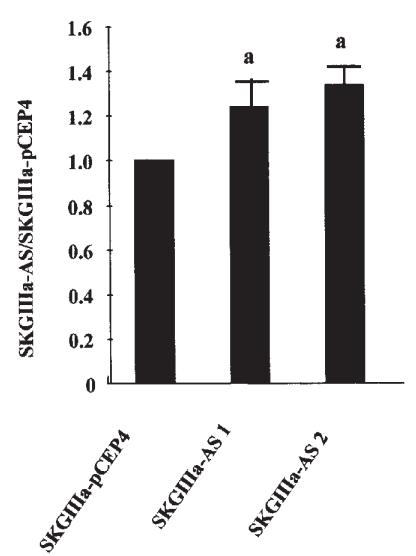

B

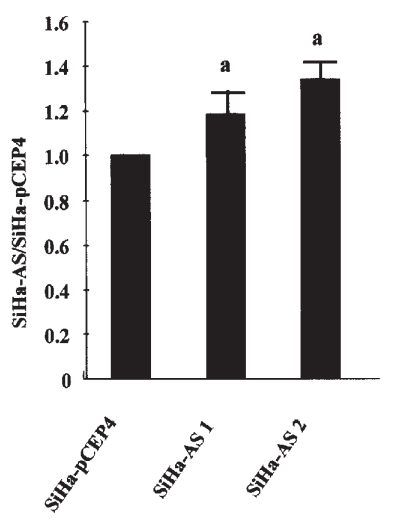

Figure 5. Effects of SCCA2 suppression on cell migration activity. Migration activity of SKG IIIa and SiHa transfected with antisense SCCA2 or an empty vector (control) was measured, as described in Materials and methods. The transfected cells and the control cells ratio is shown as mean $\pm \mathrm{SD}$ of three independent experiments. (A) SKG IIIa cells transfected with antisense SCCA2 cDNA (SKG IIIa-AS1, SKG IIIa-AS2) or the empty vector (control; SKG IIIa-pCEP4). (B) SiHa cells transfected with antisense SCCA2 cDNA (SiHa-AS1, SiHa-AS2) or the empty vector (control; SiHa-pCEP4). ${ }^{\mathrm{a} p}<0.05$ vs. anti-E-cadherin $\mathrm{Ab}(-)$.

of the parent cells (SKG IIIa and $\mathrm{SiHa}$ ) with the antibody against E-cadherin to the cells without the antibody in the non-coated chambers. The cell migration activity was also significantly increased by anti-E-cadherin antibody treatment in both SKG IIIa cells (Fig. 6A) and SiHa cells (Fig. 6B).

\section{Discussion}

The present study showed that suppression of SCCA2 expression promoted cell invasion and cell migration with the decreased expression of E-cadherin, and that blockage of 

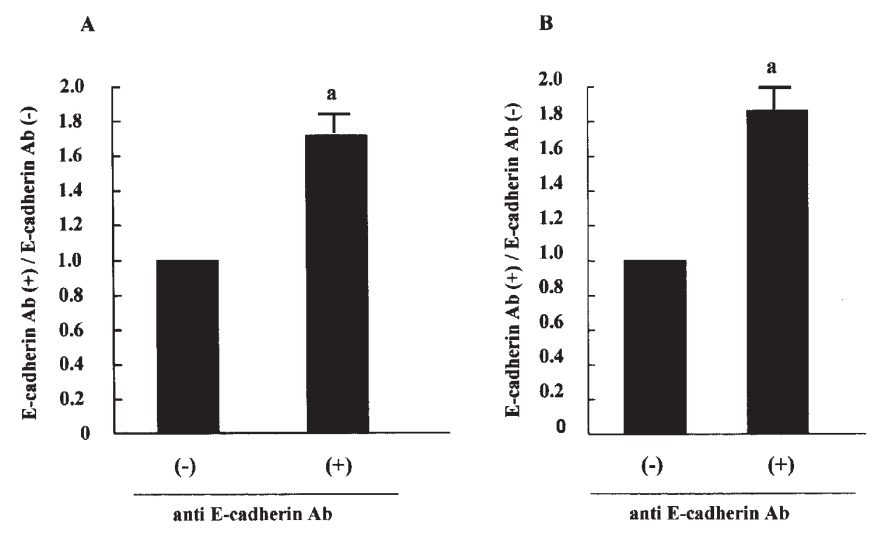

Figure 6. Effects of blockage of E-cadherin function on cell migration activity. Migration activity of SKG IIIa and SiHa with or without anti-E-cadherin antibody was investigated, as described in Materials and methods. The migrated cells were counted under a phase contrast microscope. The cells with anti-E-cadherin antibody and without antibody ratio is shown as mean \pm SD of three independent experiments. (A) SKG IIIa (B) $\mathrm{SiHa}{ }^{\mathrm{a}} \mathrm{p}<0.01$ vs. SKG IIIa or SiHa anti-E-cadherin Ab (-).

E-cadherin function by anti-E-cadherin antibody treatment inhibited cell invasion and cell migration. These findings strongly suggest that SCCA regulates cancer cell behavior through alternation in E-cadherin expression. E-cadherin is one of the most important adhesion molecules in the epithelium that mediate cell to cell contact and maintain the epithelial layer. A number of reports have demonstrated that the loss of E-cadherin plays an important role in the progression of squamous cell carcinoma and down-regulation of this protein is associated with metastasis of squamous cell carcinoma (14,21-24). Detachment of cancer cells from the primary tumor is the first step of invasion and metastasis. The E-cadherin function is suppressed, resulting in the detachment of cancer cells. After detachment from the primary tumor, cancer cells migrate, attach to vessels, and then move to other organs through blood and lymph fluid flow. In the case of the cancer cells with suppressed SCCA2, i.e. malignant transformation, loss of E-cadherin caused by the decrease in SCCA induces the detachment of cancer cells, which in turn promotes cell invasion and migration. Our unpublished data may support these results; briefly, decreased SCCA2 expression, as well as decreased E-cadherin expression, which are evaluated by immunohistochemistry, was closely associated with high incidence of lymph node metastasis, suggesting that SCCA is an important factor for lymph node metastasis. Moreover, SCCA is immunohistochemically strongly positive in welldifferentiated squamous cell carcinoma cells (25). E-cadherin also strongly expresses in well-differentiated squamous cell carcinoma cells, whereas it is negative in poorly differentiated squamous cell carcinoma cells (14).

Since matrix metalloproteinases (MMP) are also well known factors in cancer cell invasion and metastasis, we examined a possibility that SCCA regulates cell invasion and cell migration via MMP. We therefore, investigated the expression levels of MMP-2 and MMP-9 in the clones used in this study. However, there was no significant difference in MMP-2 and MMP-9 levels between any of the clones (data not shown). Iwasaki et al also reported that suppression of
E1AF, a transcriptional factor, stimulated cancer cell invasion without affecting MMP expression (26).

It is of interest to note that SCCA is expressed not only in squamous cell carcinoma tissues but also in normal squamous epithelium (15). SCCA mRNA expression is mainly observed in the basal and parabasal layers of normal squamous epithelium by in situ hybridization (16). E-cadherin is also expressed in the basal and parabasal layers (17). Therefore, there seems to be a close relationship in the formation of squamous epithelium via cell-cell adhesion between SCCA and E-cadherin.

In conclusion, the present study suggests that SCCA2 regulates cancer cell invasion and migration via E-cadherin expression. The present study provides a new insight into understanding the mechanism for malignant behavior of cancer cells. It may be also possible to control cancer cell invasion and metastasis by molecular targeting therapy with suppression of SCCA2 in the near future.

\section{Acknowledgements}

We thank Dr Shiro Nozawa for providing SKG IIIa cells and Dr Gary A. Silverman for presenting the specific antibodies for SCCA. This work was supported by a Grant-in-Aid for Scientific Research on Priority Areas (C-2) from the Ministry of Education, Science, Sports and Culture (12218224, 16591664), and by a Grant-in-Aid for Second Term Comprehensive 10-year Strategy for Cancer Control from the Ministry of Health and Welfare (H12-Gan-025), Japan.

\section{References}

1. Kato $\mathrm{H}$ and Torigoe $\mathrm{T}$ : Radioimmunoassay for tumour antigen of human cervical squamous cell carcinoma. Cancer 40: 1621-1628, 1977

2. Kato H, Tamai K, Morioka H, Nagai M, Nagaya T and Torigoe T: Prognostic significance of the tumour antigen TA-4 in squamous cell carcinoma of the uterine cervix. Am J Obstet Gynecol 145: 350-354, 1983.

3. Maruo T, Shibata K, Kimura A, Hoshina A and Mochizuki M: Tumour-associated antigen, TA-4, in the monitoring of the effects of therapy for squamous cell carcinoma of the uterine cervix. Cancer 59: 302-308, 1985.

4. Brioschi PA, Bischof P, Delafosse C and Krauer F: Squamouscell carcinoma antigen (SCC-A) values related to clinical outcome of pre-invasive and invasive cervical carcinoma. Int $\mathrm{J}$ Cancer 47: 376-379, 1991.

5. Schneider SS, Schick C, Fish KE, Miller E, Pena JC, Treter SD, Hui SM and Silverman GA: A serine proteinase inhibitor locus at $18 \mathrm{q} 21.3$ contains a tandem duplication of the human squamous cell carcinoma antigen gene. Proc Natl Acad Sci USA 92: 3147-3151, 1995.

6. Kuwano A, Kondo I, Kishi F, Suminami Y and Kato H: Assignment of the squamous cell carcinoma antigen locus (SCC) to $18 \mathrm{q} 21$ by in situ hybridization. Genomics 30: 626, 1995.

7. Suminami Y, Kishi F, Sekiguchi K and Kato H: Squamous cell carcinoma antigen is a new member of the serine protease inhibitors. Biochem Biophys Res Commun 181: 51-58, 1991.

8. Nawata S, Tsunaga N, Numa F, Tanaka T, Nakamura K and Kato $\mathrm{H}$ : Serine protease inhibitor activity of recombinant squamous cell carcinoma antigen towards chymotrypsin, as demonstrated by sodium dodecyl sulfate-polyacrylamide gel electrophoresis. Electrophoresis 16: 1027-1030, 1995.

9. Nawata S, Nakamura K, Tanaka T, Numa F, Suminami Y, Tsunaga N, Kakegawa $\mathrm{H}$, Katsunuma $\mathrm{N}$ and Kato $\mathrm{H}$ : Electrophoretic analysis of the 'cross-class' interaction between novel inhibitory serpin, squamous cell carcinoma antigen-1 and cystein proteinases. Electrophoresis 18: 784-789, 1997. 
10. Schick C, Kamachi Y, Bartuski AJ, Çataltepe S, Schechter NM, Pemberton PA and Silverman GA: Squamous cell carcinoma antigen 2 is a novel serpin that inhibits the chymotrypsin-like proteinases cathepsin $\mathrm{G}$ and mast cell chymase. J Biol Chem 17: 1849-1855, 1997.

11. Schick C, Pemberton PA, Shi GP, Kamachi Y, Çataltepe S, Bartuski AJ, Cornstein ER, Brömme D, Chapman HA and Silverman GA: Cross-class inhibition of the cysteine proteinases cathepsin K, L, S by the serpin squamous cell carcinoma antigen 1: a kinetic analysis. Biochemistry 37: 5258-5266, 1998.

12. Suminami Y, Nagashima S, Vujanovic NL, Hirabayashi K, Kato $\mathrm{H}$ and Whiteside TL: Inhibition of apoptosis in human tumour cells by the tumour-associated serpin, SCC antigen-1. Br J Cancer 82: 981-989, 2000.

13. Murakami A, Suminami Y, Hirakawa H, Nawata S, Numa F and Kato H: Squamous cell carcinoma antigen suppresses radiation-induced cell death. Br J Cancer 84: 851-858, 2001.

14. Schipper JH, Frixen UH, Behrens J, Unger A, Jahnke K and Birchmeier W: E-cadherin expression in squamous cell carcinomas of head and neck: inverse correlation with tumour dedifferentiation and lymph node metastasis. Cancer Res 51: 6328-6337, 1991.

15. Murakami A, Suminami Y, Sakaguchi Y, Nawata S, Numa F, Kishi $\mathrm{F}$ and Kato $\mathrm{H}$ : Specific detection and quantitation of $S C C$ antigen 1 and $S C C$ antigen 2 mRNAs by fluorescence-based asymmetric semi-nested reverse transcription PCR. Tumour Biol 21: 224-234, 2000.

16. Takeshima N, Suminami Y, Takeda O, Abe H, Okuno N and Kato $\mathrm{H}$ : Expression of mRNA of SCC antigen in squamous cells. Tumour Biol 13: 338-342, 1992.

17. De Boer CJ, van Dorst E, van Krieken H, Jansen-van Rhijn CM, Warnaar SO, Fleuren GJ and Litvinov SV: Changing roles of cadherins and catenins during progression of squamous intraepithelial lesions in the uterine cervix. Am J Pathol 155: 505-515, 1999.

18. Nozawa S, Kojima M, Tukazaki K, Sakayori M, Iizuka R and Kagiyama N: In vitro and in vivo induction of squamous cell carcinoma antigen (SCC) in a uterine cervical cancer cell line (SKG IIIa) with peplomycin and sodium butyrate. Asia-Oceania J Obstet Gynecol 16: 153-160, 1990.
19. Sueoka K, Nawata S, Nakagawa T, Murakami A, Takeda O, Suminami $\mathrm{Y}$, Kato $\mathrm{H}$ and Sugino N: Tumor-associated serpin, squamous cell carcinoma antigen stimulates matrix metalloproteinase-9 production in cervical squamous cell carcinoma cell lines. Int J Oncol 27: 1345-1353, 2005

20. Hirakawa H, Nawata S, Sueoka K, Murakami A, Takeda O, Numa F, Kato H and Sugino N: Regulation of squamous cell carcinoma antigen production by E-cadherin mediated cell-cell adhesion in squamous cell carcinoma cell line. Oncol Rep 11: 415-419, 2004.

21. Shiozaki H, Tahara H, Oka H, Miyata M, Kobayashi K, Tamura S, Iihara K, Doki Y, Hirano S, Takeichi M and Mori T: Expression of immunoreactive E-cadherin adhesion molecules in human cancers. Am J Pathol 139: 17-23, 1991.

22. Kadowaki T, Shiozaki H, Inoue M, Tamura S, Oka H, Doki Y, Iihara K, Matsui S, Iwazawa T, Nagafuchi A, Tsukita S and Mori T: E-cadherin and $\alpha$-catenin expression in human esophageal cancer. Cancer Res 54: 291-296, 1994.

23. Vessey CJ, Wilding J, Folarin N, Hirano S, Takeichi M, Soutter P, Stamp GWH and Pignatelli M: Altered expression and function of E-cadherin in cervical intraepithelial neoplasia and invasive squamous cell carcinoma. J Pathol 176: 151-159, 1995.

24. Carico E, Atlante M, Bucci B, Nofroni I and Vecchione A: Ecadherin and $\alpha$-catenin expression during tumour progression of cervical carcinoma. Gynecol Oncol 80: 156-161, 2001.

25. Cataltepe S, Schick C, Luke CJ, Pak SCO, Goldfarb D, Chen P, Tanasiyevic MJ, Posner MR and Silverman GA: Development of specific monoclonal antibodies and a sensitive discriminatory immunoassay for the circulating tumour markers SCCA1 and SCCA2. Clin Chim Acta 295: 107-127, 2000.

26. Iwasaki M, Nishikawa A, Akutagawa N, Fujimoto T, Teramoto M, Sakaguchi Y, Kato H, Ito M, Yoshida K and Kudo R: E1AF/ PEA3 reduces the invasiveness of SiHa cervical cancer cells by activating serine proteinase inhibitor squamous cell carcinoma antigen. Exp Cell Res 299: 525-532, 2004. 